\title{
EFEITOS DA INOCULAÇÃO DE FUNGOS MICORRÍZICOS ARBUSCULARES E DA APLICAÇÃO DE FÓSFORO NO ESTABELECIMENTO DE FORRAGEIRAS EM SOLO DEGRADADO ${ }^{1}$
}

\author{
MARCO AURÉLIO CARBONE CARNEIRO², JOSÉ OSWALDO SIQUEIRA ${ }^{3}$, \\ NILTON CURI ${ }^{3}$ e FÁTIMA MARIA SOUZA MOREIRA ${ }^{3}$
}

\begin{abstract}
RESUMO - Avaliaram-se os efeitos de doses de fósforo (0, 50, 100, 200 e $400 \mathrm{mg} \mathrm{kg}^{-1}$ de solo) e da inoculação de uma mistura de fungos micorrízicos arbusculares (FMAs) (Glomus etunicatum, Glomus clarum, Gigaspora margarita e Acaulospora scrobiculata) no acúmulo de nitrogênio, fósforo e no crescimento do estilosantes (Stylosanthes guianensis Aubl. sw.), capim-gordura (Melinis minutiflora Beauv.), capim-braquiária (Brachiaria decumbens Stapf.) e do cultivo misto com estilosantes e capim-gordura em solo degradado. O experimento foi desenvolvido em casa de vegetação, sendo realizados três cortes da parte aérea das plantas, com intervalo de 150 dias. As raízes foram coletadas no último corte. Verificou-se, na matéria seca da parte aérea (MSPA) e de raízes (MSR), no acúmulo de $\mathrm{N}$ e de $\mathrm{P}$, resposta positiva a doses crescentes de $\mathrm{P}$ em todas as espécies estudadas. A inoculação de FMAs aumentou a MSPA, MSR e o acúmulo de $\mathrm{N}$ e $\mathrm{P}$, e causou diferenciação da magnitude deste aumento entre as espécies. $\mathrm{O}$ estilosantes apresentou baixa colonização ( $4 \%$ em média), porém foi muito responsivo à inoculação de FMAs. O efeito da aplicação de $\mathrm{P}$ no acúmulo de N na MSPA foi favorecido pela inoculação de FMAs. O uso do capim-gordura, que não depende dos FMAs para alcançar o estabelecimento adequado, pode ser uma estratégia para a revegetação de solos degradados sem inoculação de FMAs.
\end{abstract}

Termos para indexação: revegetação, Stylosanthes guianensis, Brachiaria decumbens, Melinis minutiflora.

EFFECTS OF ARBUSCULAR MYCORRHIZAL FUNGI INOCULATION AND PHOSPHORUS APPLICATION ON FORAGE CROPS ESTABLISHMENT IN A DEGRADED SOIL

\begin{abstract}
The effects of increasing doses of phosphorus $\left(0,50,100,200\right.$ and $400 \mathrm{mg}$ of $\mathrm{P} \mathrm{kg}^{-1} \mathrm{of}^{-}$ soil) and of inoculation with a mixture of arbuscular mycorrhizal fungi (AMF) upon accumulation of nitrogen and phosphorus and growth of Stylosanthes guianensis, Brachiaria decumbens, Melinis minutiflora and Stylosanthes guianensis - Melinis minutiflora mixed cultivation were evaluated in soil degraded by hydro-electrical dam construction. The experiment was conducted under greenhouse, and three cuts of the aerial part of the plants were performed with an interval of 150 days, and roots were collected only at the last cut. Shoot and root and plant accumulation of $\mathrm{N}$ and $\mathrm{P}$ in all species responded positively to $\mathrm{P}$. Inoculation with AMF also increased all the above mentioned parameters. The S. guianensis presented low AMF colonization (mean of 4\%), but it was very responsive to inoculation with these fungi. The effects of $\mathrm{P}$ application upon $\mathrm{N}$ accumulation on dry matter yield were also favored by inoculation with AMF. The use of M. minutiflora, that does not depend upon AMF for achieving adequate growth, can be a reasonable strategy for revegetation of degraded soils, without artificial inoculation with AMF.
\end{abstract}

Index terms: revegetation, Stylosanthes guianensis, Brachiaria decumbens, Melinis minutiflora.

\footnotetext{
${ }^{1}$ Aceito para publicação em 30 de outubro de 1998.

Extraído da Dissertação de Mestrado apresentada pelo primeiro autor à Universidade Federal de Lavras (UFLA). Trabalho parcialmente financiado pela CEMIG

${ }^{2}$ Eng. Agr., M.Sc., Doutorando, Dep. de Ciência do Solo, Universidade Federal de Lavras, Caixa Postal 37, CEP 37200-000 Lavras, MG. Bolsista do CNPq. E-mail: mcarbone@ufla.br

${ }^{3}$ Eng. Agr., Ph.D., Dep. de Ciência do Solo, UFLA. Bolsista do CNPq.
}

\section{INTRODUÇÃO}

Solo degradado é aquele que apresenta declínio da sua qualidade ou capacidade produtiva, causada pelo uso incorreto, como o desmatamento, monocultura intensiva, uso indiscriminado de produtos químicos, mineração e construção civil, resultando em degradação física (compactação e 
erosão), química (diminuição de nutrientes e acúmulo de produtos tóxicos) e biológica (diminuição da atividade e diversidade da microfauna e flora do solo) (Lal \& Stewart, 1989).

Estudos sobre a revegetação de áreas perturbadas mostram que a introdução de espécies herbáceas facilita a interação entre os fatores químicos, físicos e biológicos do solo (Luchesi et al., 1992; Fisher et al., 1994). Estas espécies, dado o seu rápido crescimento vegetativo e grande volume de raízes, protegem o solo contra o impacto direto das gotas de chuva, evitando a desestruturação do solo, aumentam a taxa de infiltração de água, reduzindo o escorrimento superficial, diminuindo o assoreamento dos rios e barragens, e aumentam o teor de matéria orgânica, fixando $\mathrm{C}$ no solo e incrementando a atividade microbiana.

Para garantir o sucesso da ação antrópica na recuperação de áreas degradadas é necessário selecionar espécies vegetais rústicas, tolerantes a períodos secos e à baixa fertilidade, e capazes de produzir grande quantidade de matéria orgânica e sementes viáveis (Medeiros et al., 1987; Siqueira et al., 1994). A braquiária, o capim-gordura e o estilosantes são espécies que apresentam estes atributos (Botrel, 1983; Alves, 1988), representando grandes possibilidades para programas de recomposição da vegetação.

Solos que perderam sua camada arável apresentam quantidade reduzida de propágulos de microrganismos benéficos para as plantas, tornando-se a introdução destes muito importante do ponto de vista ecológico, e de grande interesse tecnológico. A maioria das espécies vegetais forma associações mutualistas entre certos fungos de solo e raízes, as quais denominam-se micorrizas. Estas incrementam a absorção de nutrientes, em especial de $\mathrm{P}$, e a tolerância das plantas a diversos tipos de estresses, influenciando seu crescimento e reprodução (Siqueira, 1994). Além disso, as leguminosas, como o estilosantes, formam também simbiose com Bradyrhizobium, bactéria fixadora de $\mathrm{N}$ atmosférico que pode contribuir assim para o enriquecimento do solo com N. Portanto, os efeitos da re-introdução destes organismos nos solos degradados devem ser avaliados, como também sua relação com a fertilidade do solo.

No presente trabalho avaliaram-se os efeitos da inoculação de fungos micorrízicos arbusculares (FMAs) e das doses crescentes de P no crescimento vegetativo e no acúmulo de $\mathrm{Ne} \mathrm{P}$ do capim-braquiária, capim-gordura, estilosantes e do cultivo misto entre o capim-gordura e estilosantes, em solo degradado.

\section{MATERIAL E MÉTODOS}

$\mathrm{O}$ estudo foi realizado em casa de vegetação do Departamento de Ciência do Solo da Universidade Federal de Lavras, MG, com solo degradado. O solo é remanescente de um Latossolo Vermelho-Escuro (LE), do qual foi retirado material (sólum) para a construção de uma hidrelétrica (área de empréstimo) há mais de trinta anos e atualmente ainda está desprovido de vegetação.

Amostra do solo foi secada ao ar e peneirada, e recebeu calcário dolomítico, para elevar a saturação por bases para 65\% (Raij, 1991). Após a calagem, o solo ficou incubado por 15 dias, sendo então adubado com cinco doses de $\mathrm{P}\left(0,50,100,200\right.$ e $400 \mathrm{mg} \mathrm{kg}^{-1}$ de solo), aplicado na forma de superfosfato triplo. Após a calagem e a adubação, o solo apresentou $\mathrm{pH}$ em água $=6,5$; $\mathrm{K}=16 \mathrm{mg} \mathrm{dm}^{-3} ; \mathrm{Ca}=13 \mathrm{mmol}_{\mathrm{c}} \mathrm{dm}^{-3} ; \mathrm{Mg}=7 \mathrm{mmol}_{\mathrm{c}} \mathrm{dm}^{-3}$; $\mathrm{Al}=0 ; \mathrm{H}=12 \mathrm{mmol}_{\mathrm{c}} \quad \mathrm{dm}^{-3} ; \mathrm{S}=20 \mathrm{mmol}_{\mathrm{c}} \mathrm{dm}^{-3}$; $\mathrm{t}=20 \mathrm{mmol}_{\mathrm{c}} \mathrm{dm}^{-3}$; e $\mathrm{T}=32 \mathrm{mmol}_{\mathrm{c}} \mathrm{dm}^{-3}$. Os teores de $\mathrm{P}$ (Mehlich-1) após a aplicação das doses de superfosfato triplo foram de 6, 15, 22, 63 e $155 \mathrm{mg} \mathrm{dm}^{-3}$ de P. Estes valores correlacionaram-se positivamente com a quantidade de $\mathrm{P}$ aplicado $(\mathrm{y}=-5,4+0,384 \mathrm{x} ; \mathrm{y}=\mathrm{P}$ recuperado; $\mathrm{x}=\mathrm{P}$ aplicado; $\left.\mathrm{R}^{2}=0,98^{* *}\right)$.

Utilizaram-se vasos com capacidade para $1,8 \mathrm{~kg}$ de solo, onde foram semeadas as seguintes espécies: estilosantes (Stylosanthes guianensis Aubl. Sw.), capim-gordura (Melinis minutiflora Beauv.), capim-braquiária (Brachiaria decumbens Stapf.) e o cultivo misto com estilosantes e capim-gordura, tendo sido submetidas aos tratamentos (doses de $\mathrm{P}$ e inoculação) em delineamento inteiramente casualizado, em esquema fatorial $5 \times 2$, com cinco repetições, e cada espécie ou mistura foi considerada um experimento.

As sementes foram colocadas diretamente nos vasos com os tratamentos, quando foram então inoculadas com fungos micorrízicos. Após atingirem $5 \mathrm{~cm}$ de altura, procedeu-se um desbaste, deixando 2 plantas/vaso, e no tratamento cultivo misto, cada espécie teve 1 planta/vaso. Utilizou-se como inóculo uma mistura de fungos 
micorrízicos arbusculares (FMAs) composta de Glomus etunicatum Becker \& Gerdemann, Glomus clarum Nicolson \& Sckenck, Gigaspora margarita Becker \& Hall e Acaulospora scrobiculata Trappe, obtidos de vasos de cultivo com capim-braquiária. O inóculo constou de raízes picadas, esporos, hifas e outros propágulos, aplicando-se $5 \mathrm{~mL}$ por vaso, suficientes para fornecer aproximadamente 270 esporos. Visando equilibrar a microbiota entre os tratamentos infectados dos que não haviam sido infectados, estes receberam $5 \mathrm{~mL}$ de um filtrado do inóculo isento de propágulos de fungos MAs, obtido da suspensão de $50 \mathrm{~cm}^{3}$ de substrato dos vasos de cultivo em 15 litros de água e filtragem em papel de filtro. No solo não infectado foram recuperados três esporos de FMAs por $50 \mathrm{~mL}$ de solo. No tratamento com estilosantes e no cultivo misto, procedeu-se também à inoculação de Bradyrhizobium sp., utilizando $5 \mathrm{~mL} /$ vaso de uma cultura das estirpes BR 446 e BR 502 em meio Norris contendo $1,4 \times 10^{5}$ bactérias $/ \mathrm{mL}$. Para a contagem de esporos, estes foram extraídos por peneiramento úmido do solo, seguido de centrifugações em água e sacarose a $50 \%$ (Gerdemann \& Nicolson, 1963) e contados em microscópio estereoscópio (40x).

O experimento foi conduzido por 450 dias, sendo realizados três cortes, a $4 \mathrm{~cm}$ do solo nos dois primeiros e no último no colo da planta, com intervalo de 150 dias entre cada corte. A umidade do solo foi ajustada para atingir $60 \%$ do volume total de poros, e assim mantida por meio de pesagens periódicas, durante todo o experimento. No capim-braquiária e no capim-gordura, foram realizadas três aplicações de $\mathrm{N}$, totalizando $150 \mathrm{mg} \mathrm{vaso}^{-1}$, a saber: a primeira, no início do experimento, e as demais, após cada corte das plantas. O material da parte aérea foi lavado e secado em estufa a $80^{\circ} \mathrm{C}$, até peso constante, obtendo-se o peso da matéria seca da parte aérea de cada corte. O material foi moído em moinho tipo Wiley, e utilizado para análises dos teores de nutrientes nos extratos dos tecidos (Hunter, 1975) e cálculos das quantidades acumuladas. Os teores de $\mathrm{P}$ foram determinados por colorimetria (azul de molibdênio) e $\mathrm{N}$ por digestão com $\mathrm{H}_{2} \mathrm{SO}_{4}$ (Sarruge \& Haag, 1974).

Após o último corte, as raízes foram separadas do solo com o auxílio de uma peneira, lavadas, sendo retirado $1 \mathrm{~g}$ de raízes finas para avaliação da colonização micorrízica, e o restante foi seco em estufa. Para a avaliação da colonização, as amostras foram clarificadas (Kormanik \& McGraw, 1982) e avaliadas em placa quadriculada (Giovannetti \& Mosse, 1980). Ao final do experimento, retiraram-se também amostras de solo para extração (Gerdemann \& Nicolson, 1963), contagem e identificação dos esporos (Schenck \& Pérez, 1987). A nodulação do estilosantes foi avaliada por um sistema de notas, atribuindo-se valores menores que 1 , quando as plantas apresentavam menos de 20 nódulos planta ${ }^{-1}$; nota $2 \mathrm{com}$ 20 a 50 nódulos planta ${ }^{-1}$; e nota 3 , referente a plantas com mais de 50 nódulos planta ${ }^{-1}$.

Os dados foram submetidos à análise de variância e teste de médias, pelo programa estatístico Sanest (Zonta et al., 1984), e as regressões foram obtidas pelo programa Tablecurve (Jandel Scientific Corporation), obtendo-se as equações de melhor ajuste e com teste de F significativo.

\section{RESULTADOS E DISCUSSÃO}

O estilosantes apresentou, na matéria seca da parte aérea (MSPA), elevada resposta positiva a $\mathrm{P}$ e à inoculação com FMAs, nos três cortes, sendo os efeitos das doses de $\mathrm{P}$ diferenciados nos cortes (Fig. 1).

$\mathrm{Na}$ produção de MSPA máxima do primeiro corte, a inoculação propiciou um aumento de $307 \%$, sendo que no segundo corte este aumento foi de menor magnitude - cerca de $22 \%$-, devido à maior produção de plantas não-inoculadas. No terceiro corte, a inoculação teve efeito até a dose $270 \mathrm{mg} \mathrm{kg}^{-1}$ de $\mathrm{P}$, apresentando efeito depressivo em doses superiores (Fig. 1c).

A aplicação de apenas $50 \mathrm{mg} \mathrm{kg}^{-1}$ de $\mathrm{P}$, juntamente com a inoculação de FMAs, oferece condições favoráveis para o estabelecimento do estilosantes neste solo, corroborando resultados com esta espécie em outros solos (Lambais, 1987; Saif, 1987; Alves, 1988).

$\mathrm{Na}$ braquiária, o $\mathrm{P}$ teve pequeno aumento na MSPA nos três cortes, e efeito positivo da inoculação apenas no primeiro corte, sendo este efeito muito pequeno. No terceiro corte, a inoculação foi depressiva a partir da dose $27 \mathrm{mg} \mathrm{kg}^{-1}$ de $\mathrm{P}$ (Fig. 1f). Considerando-se a produção máxima, a inoculação aumentou a MSPA da braquiária em 30\% no primeiro corte. A MSPA foi maior no terceiro corte, provavelmente devido à colonização das raízes pelos fungos indígenas ao longo do tempo. Assim como foi verificado no estilosantes, a aplicação de $50 \mathrm{mg} \mathrm{kg}^{-1}$ de P é suficiente para o estabelecimento do capim-braquiária neste solo.

A aplicação de $\mathrm{P}$ aumentou a MSPA do capim-gordura, nos três cortes. Em relação à inoculação de FMAs isto não ocorreu, exceto no terceiro corte (Fig. 1), o que indica que o capim-gordura é uma espécie pouco influenciada pela micorrização.

Pesq. agropec. bras., Brasília, v.34, n.9, p.1669-1677, set. 1999 


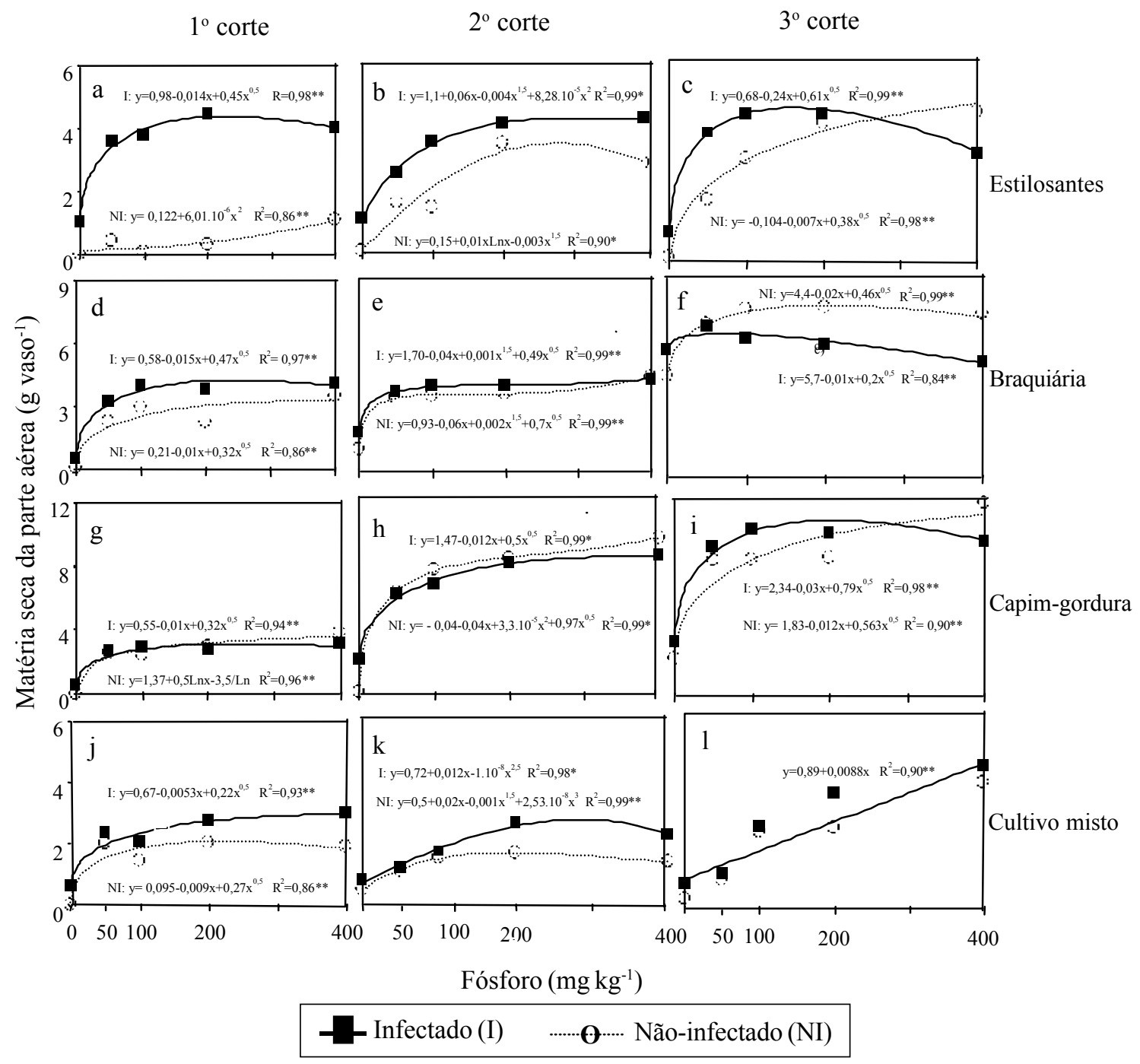

FIG. 1. Matéria seca da parte aérea nos três cortes realizados nas diferentes plantas estudadas em função da inoculação micorrízica e dose de fósforo (* e ** regressão significativa pelo teste $\mathrm{F}$ a $5 \%$ e $1 \%$, respectivamente).

No cultivo misto, houve resposta positiva quanto a ambos os fatores, nos dois primeiros cortes, e a resposta a $\mathrm{P}$ foi linear no terceiro corte (Fig. 1). Verificou-se menor produção de MSPA total em relação ao estilosantes e ao capim-gordura, plantados separadamente.

No cultivo separado, foram colhidas duas plantas, enquanto no cultivo misto cada espécie contribuiu com uma planta; além deste fato, verificou-se pequeno crescimento do estilosantes em relação ao cultivo separado, e ocorreu redução de pelo menos $50 \%$ da MSPA. Além disso, não foi aplicado $\mathrm{N}$ no cultivo misto, e o $\mathrm{N}$ fixado pelo estilosantes parece não ter sido suficiente para atender à demanda de $\mathrm{N}$ das plantas neste tratamento.

Com base nas respostas das plantas aos fatores estudados, determinou-se, através das regressões, o impacto da inoculação na redução do requerimento 
externo de P (REP) para atingir $90 \%$ da produção máxima de matéria seca da parte aérea. Isto foi superior a $76 \%$ no estilosantes, $73 \%$ em braquiária, e $55 \%$ no cultivo misto no primeiro corte e aos valores da mesma ordem no segundo corte. Quanto ao capim-gordura no primeiro e segundo cortes, estes efeitos são desprezíveis. No terceiro corte, os efeitos foram ainda observados no estilosantes, e ocorreram também em capim-gordura.

A matéria seca de raízes (MSR) só foi avaliada ao final do experimento, quando houve comportamento diferenciado entre as espécies. Verificou-se efeito significativo $(\mathrm{P} \leq 0,05)$ da inoculação no estilosantes, braquiária e capim-gordura, e a inoculação foi depressiva para capim-gordura (Tabela 1).

$\mathrm{O} P$ só teve efeito no cultivo misto $\left(\mathrm{y}=1,46+0,0184 \mathrm{x}-0,00004 \mathrm{x}^{2} ; \mathrm{R}^{2}=0,70^{* *} ; \mathrm{x}=\right.$ dose de $\mathrm{P} ; \mathrm{y}=\mathrm{MSR}$ ) e no capim-gordura, apresentando, neste último, interação significativa com a inoculação (plantas infectadas: $\mathrm{y}=2,36-0,001 \mathrm{x}^{1,5}+0,28 \mathrm{x}^{0,5}-0,0002 / \mathrm{x}^{2} ; \mathrm{R}^{2}=0,99 * * \mathrm{e}$ plantas não-infectadas: $\mathrm{y}=0,699+0,333 \mathrm{x}^{0,5}$; $\mathrm{R}^{2}=0,88^{* *} ; \mathrm{x}=$ dose de $\left.\mathrm{P} ; \mathrm{y}=\mathrm{MSR}\right)$. Os efeitos dos tratamentos na massa de raízes foram bem menores que os da parte aérea.

TABELA 1. Médias gerais da matéria seca de raízes (MSR), colonização micorrízica (Col.) e densidade de esporos (DE) no solo, nas espécies vegetais (Esp.), em função da inoculação de fungos micorrízicos arbusculares (Inoc.) e efeito de doses crescentes de $\mathbf{P}^{\mathbf{1}}$.

\begin{tabular}{llllllll}
\hline Esp. & Inoc. & MSR & Efeito P & Col. & Efeito P & DE & Efeito P \\
\hline Est. & I & $0,95 \mathrm{a}$ & $\mathrm{ns}$ & $4 \mathrm{a}$ & $\mathrm{ns}$ & $545 \mathrm{a}$ & $\mathrm{ns}$ \\
& NI & $0,50 \mathrm{~b}$ & $\mathrm{~ns}$ & $2 \mathrm{a}$ & $\mathrm{ns}$ & $107 \mathrm{~b}$ & $\mathrm{~ns}$ \\
Braq. & $\mathrm{I}$ & $4,14 \mathrm{a}$ & $\mathrm{ns}$ & $51 \mathrm{a}$ & $\mathrm{ns}$ & $165 \mathrm{a}$ & $\mathrm{ns}$ \\
& NI & $3,29 \mathrm{~b}$ & $\mathrm{~ns}$ & $57 \mathrm{a}$ & $\mathrm{ns}$ & $17 \mathrm{~b}$ & $\mathrm{~ns}$ \\
C.G. & $\mathrm{I}$ & $3,64 \mathrm{~b}$ & $\mathrm{Eq} 1$ & $15 \mathrm{a}$ & $\mathrm{ns}$ & $288 \mathrm{a}$ & Eq1 \\
& NI & $4,11 \mathrm{a}$ & $\mathrm{Eq1}$ & $12 \mathrm{a}$ & $\mathrm{ns}$ & $162 \mathrm{~b}$ & Eq1 \\
C.M. & $\mathrm{I}$ & $2,59 \mathrm{a}$ & Eq2 & $9 \mathrm{a}$ & $\mathrm{ns}$ & $496 \mathrm{a}$ & Eq2 \\
& NI & $2,72 \mathrm{a}$ & & $12 \mathrm{a}$ & $\mathrm{ns}$ & $202 \mathrm{~b}$ & \\
\hline
\end{tabular}

${ }^{1}$ Médias seguidas pela mesma letra (efeito da inoculação de fungos micorrízicos arbusculares) não diferem entre si pelo teste de Tukey a 5\%; Est.: estilosantes; Braq.: braquiária; C.G.: capim-gordura; C.M.: cultivo misto; I: com inoculção; NI: sem inoculação; ns: não-significativo; Eq1: efeito da interação entre doses de P e inoculação de FMAs; Eq2: efeito de doses de P.
O estilosantes apresentou pequena produção de MSR, porém houve grande efeito da inoculação, fato já relatado em outros estudos (Lambais, 1987; Alves, 1988).

O capim-braquiária produziu em torno de 4 vezes mais raízes que o estilosantes, e também respondeu à inoculação. Apesar do efeito depressivo da inoculação, o capim-gordura foi a espécie com maior produção de MSR, atingindo até 7,0 g de MSR por vaso na dose mais elevada de $\mathrm{P}$ em plantas não-infectadas.

No cultivo misto, houve resposta positiva à aplicação de $\mathrm{P}$, e nenhum efeito da inoculação ou interação entre os fatores estudados. A produção de raízes é importante, pois além de favorecer a atividade de microrganismos heterotróficos e assim contribuir para aumentar a biomassa microbiana e recuperar a atividade biológica do solo degradado, tem papel fundamental na estruturação e em outros atributos físicos do solo (Crowell \& Boerner, 1988; Siqueira et al., 1994; Siqueira \& Saggin-Junior, 1995).

A colonização micorrízica ao término do experimento foi máxima na braquiária, e não foi influenciada pelos fatores estudados, em nenhuma espécie (Tabela 1). A ausência de diferença entre os tratamentos com e sem inoculação deve-se ao fato de o solo não ter sido fumigado. Mesmo apresentando apenas três esporos $/ 50 \mathrm{~mL}$ de solo no início do experimento, estes e outros propágulos foram suficientes para promover, durante os 450 dias de duração do experimento, colonização semelhante entre plantas infectadas e não-infectadas. Entretanto, diferenças podem ter ocorrido no início do estabelecimento das plantas, o que não foi avaliado neste estudo. Verificou-se que os efeitos da inoculação tenderam a diminuir com os cortes, indicando benefícios crescentes da colonização pelos fungos nativos do solo.

A nodulação do estilosantes isolado e em cultivo misto variou com as doses de $\mathrm{P}$ e com a inoculação com FMAs. Nas plantas infectadas com fungos MAs, verificou-se que a aplicação de $50 \mathrm{mg} \mathrm{kg}^{-1}$ de $\mathrm{P}$ proporcionou máxima nodulação (nota 3 ), enquanto plantas não-infectadas apresentaram baixa nodulação (nota $<1$ ), em todas as doses de $\mathrm{P}$. 
No cultivo misto, a nodulação foi baixa, porém plantas com FMAs apresentaram maior nodulação que plantas não-infectadas. A deficiência de P é um dos fatores limitantes para o crescimento e nodulação do estilosantes (Mosse, 1977), e a simbiose mutualista com FMAs permite que estas plantas tenham desenvolvimento e nodulação satisfatórios em solos com deficiência deste nutriente (Lambais, 1987; Alves, 1988).

A densidade de esporos no solo após o cultivo das plantas foi favorecida pela inoculação nas três espécies e no cultivo misto (Tabela 1), e foi em média máxima na presença de estilosantes inoculado, apesar da baixa colonização micorrízica desta espécie.

No estilosantes, na braquiária e no cultivo misto, as doses de $\mathrm{P}$ não influenciaram o número total de esporos recuperados no solo, enquanto no capim-gordura infectado houve interações entre os tratamentos e a densidade de esporos aumentou linearmente com as doses de $\mathrm{P}$ (plantas infectadas: $\mathrm{y}=126,8+1,08 \mathrm{x} ; \mathrm{R}^{2}=0,91 * *$ e plantas não-infectadas: $y=-4,99+7,192 x-0,051 x^{2}+0,00009 x^{3} ; R^{2}=0,99 * *$; $\mathrm{x}=$ dose de $\mathrm{P} ; \mathrm{y}=$ densidade de esporos), atingindo, nas plantas infectadas, valores de densidade próximos aos obtidos para o estilosantes. Apesar de não responder à inoculação, o capim-gordura mostrou-se bom hospedeiro para multiplicação destes fungos no solo degradado adubado com P. A composição de espécies de fungos micorrízicos no solo variou com a planta e doses de P. Nos solos infectados, Glomus etunicatum foi a espécie predominante. No solo com apenas capim-gordura, predominou a Acaulospora scrobiculata, enquanto na braquiária o Glomus occultum foi a espécie dominante. Dos fungos introduzidos, apenas G. etunicatum e A. scrobiculata foram recuperados no período estudado, o que indica certa adaptação destes ao ambiente do solo degradado, enquanto G. clarum e G. margarita esporularam fracamente nestes hospedeiros. $\mathrm{O} \mathrm{pH}$ elevado deste solo após a correção $(6,5)$ pode ter contribuído para a baixa esporulação da G. margarita, que prefere $\mathrm{pH}$ ácido (Siqueira et al., 1986). Por outro lado, a elevada esporulação do G. etunicatum é coerente com sua ocorrência generalizada nos solos da região Sudeste do Brasil (Siqueira et al., 1989). No solo não-infectado, verificou-se grande variação na composição das espécies fúngicas.
Os efeitos da aplicação de $\mathrm{P}$ e da inoculação de FMAs no acúmulo de N e P na MSPA das plantas foram bastante acentuados, conforme equações de respostas apresentadas na Tabela 2. No primeiro corte, plantas de estilosantes infectadas apresentaram maior acúmulo de $\mathrm{N}$, atingindo o máximo de $108 \mathrm{mg}_{\text {planta }}{ }^{-1}$ de $\mathrm{N}$ na dose $272 \mathrm{mg} \mathrm{kg}^{-1}$ de $\mathrm{P}$. Já as plantas não-infectadas apresentaram elevado requerimento externo de $\mathrm{P}$ e acumularam muito pouco $\mathrm{N}$, apenas $23 \mathrm{mg} \mathrm{planta}^{-1}$. Nos demais cortes, só foram verificados efeitos do $\mathrm{P}$ no acúmulo de $\mathrm{N}$ nesta espécie. Isto evidência o sinergismo entre micorriza e adubação fosfatada em solos de baixa fertilidade (Mosse, 1977; Lambais, 1987; Saif, 1987; Alves, 1988). Na braquiária, observou-se efeito de $\mathrm{P}$ em todos os cortes, porém efeito pequeno para inoculação. No capim-gordura só houve efeito de $P$, enquanto no cultivo misto a aplicação de $\mathrm{P}$ e a presença do estilosantes aumentaram o acúmulo de N na MSPA. As plantas acumularam menores quantidades de $\mathrm{P}$ do que de $\mathrm{N}$, e o acúmulo de $\mathrm{P}$ foi favorecido pela elevação da dose aplicada deste elemento e pela inoculação de FMAs. Assim como foi verificado para o N, o acúmulo de $\mathrm{P}$ foi maior nas plantas de estilosantes infectadas de FMAs no primeiro e segundo cortes. Não houve diferenças marcantes entre os cortes quanto a este parâmetro, sendo que a braquiária tendeu a acumular mais P no segundo e terceiro corte. Isto pode estar relacionado à capacidade destas espécies de se estabelecerem satisfatoriamente mesmo em solos de baixa fertilidade (Botrel, 1983).

$\mathrm{O}$ acúmulo de nutrientes na matéria seca da parte aérea é uma estratégia importante na recuperação de solos degradados, e neste contexto a inoculação de fungos micorrízicos tem papel importante, pois favorece este acúmulo nas espécies com maior grau de micotrofia (Sims, 1989; Siqueira et al., 1994). Em solos de baixa fertilidade, existe estreita relação entre a nutrição nitrogenada e a fosfatada: ambas são muito influenciadas pelos FMAs (Siqueira \& Saggin-Junior, 1995).

No solo degradado, isto não foi diferente: no primeiro corte, a quantidade total de $\mathrm{N}$ acumulada correspondente a cada $\mathrm{kg}$ de $\mathrm{P}$ aplicado no solo, na dose de $\mathrm{P}$ referente ao acúmulo máximo de $\mathrm{N}$ (Tabela 2), foi 800 vezes maior no estilosantes e 320 vezes na braquiária, nas plantas infectadas do que 
EFEITOS DA INOCULAÇÃO DE FUNGOS MICORRÍZICOS ARBUSCULARES 1675

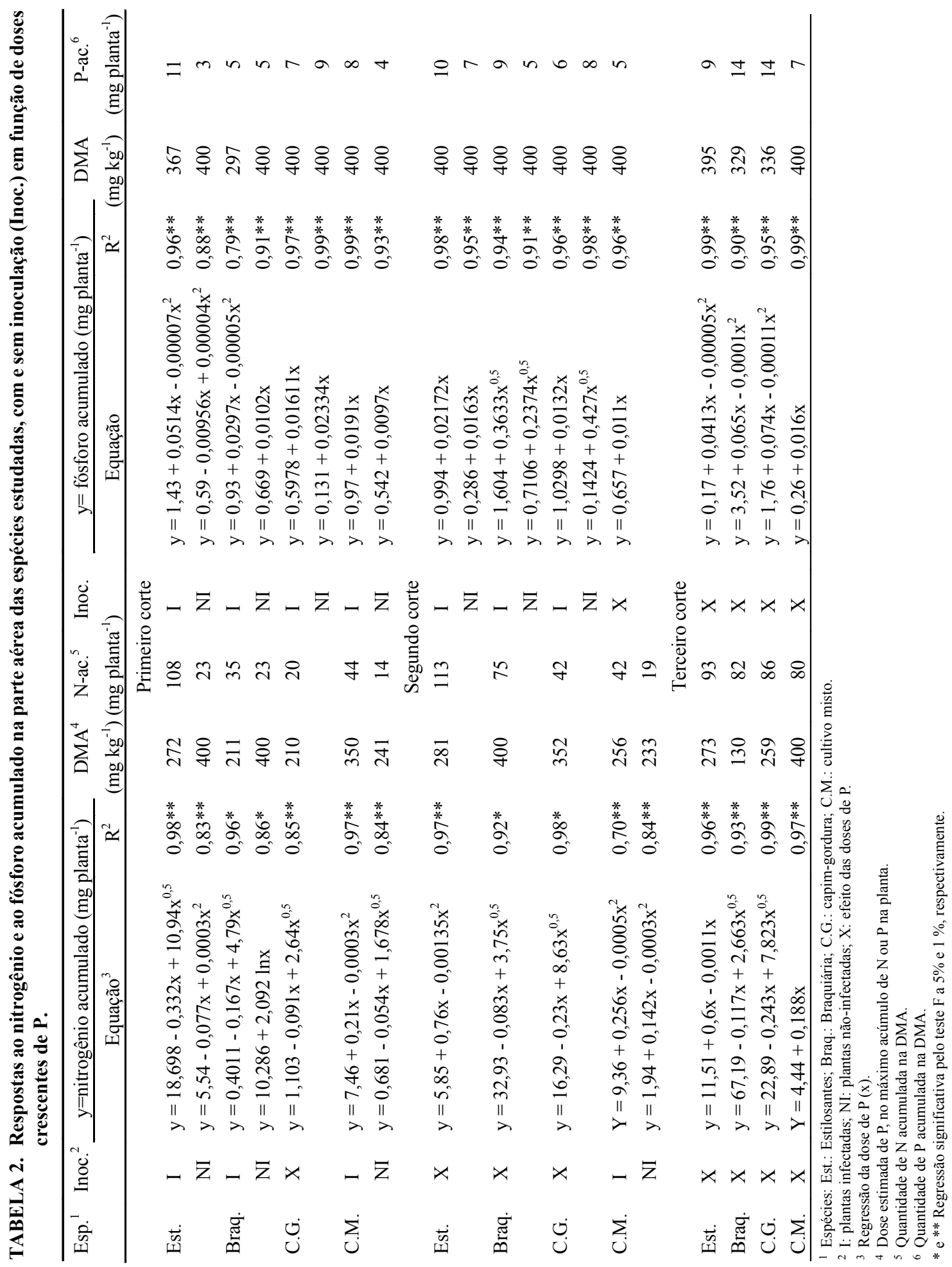


nas não-infectadas. Quando esta relação é expressa em mg de $\mathrm{N}$ por mg de $\mathrm{P}$ acumulados na MSPA, são encontrados valores de 9,8 e 7,6 no estilosantes e 7,0 e 4,6 no capim-braquiária, nas plantas infectadas e não-infectadas, respectivamente.

Estes resultados indicam que as micorrizas arbusculares são de grande importância na acumulação de $\mathrm{N}$ no solo em recuperação, mesmo quando este é revegetado com capim-braquiária, que é pouco responsivo à inoculação para a produção de massa. Como o capim-gordura comportou-se indiferentemente à inoculação, ele apresenta vantagens na introdução em áreas degradadas e que apresentam baixa densidade de propágulos de FMAs, ao contrário das espécies dependentes destes fungos, que terão menores chances de se estabelecer em solos com baixa infectividade de propágulos.

Como demonstrado em estudos anteriores, o solo em estudo apresenta severas limitações de $\mathrm{N}$ e $\mathrm{P}$ (Carneiro et al., 1995), e sabe-se que a aplicação destes nutrientes e a capacidade das plantas de absorver e de acumulá-los na fitomassa são fatores essenciais para recuperação do solo (Sims, 1989), pois ao serem incorporados no ciclo orgânico do ecossistema contribuirão para melhorar a atividade biológica e aumentar gradualmente a fertilidade e o condicionamento físico do solo em recuperação (Luchesi et al., 1992; Fisher et al., 1994).

\section{CONCLUSÕES}

1. A produção de massa seca e o acúmulo de $\mathrm{Ne}$ $\mathrm{P}$ na parte aérea do estilosantes, capim-braquiária, capim-gordura e cultivo misto, beneficiam-se da inoculação de fungos micorrízicos arbusculares e da aplicação de superfosfato, e estes efeitos são diferenciados entre as espécies e cortes.

2. A inoculação de fungos micorrízicos arbusculares e a aplicação de $\mathrm{P}$ atuam de modo sinergístico no estabelecimento destas espécies no solo estudado.

3. Os fungos micorrízicos arbusculares reduzem o requerimento externo de $\mathrm{P}$ do estilosantes, capim-braquiária e cultivo misto, e não têm efeito no capim-gordura.
4. O efeito da aplicação de P no acúmulo de $\mathrm{N}$ nas plantas é favorecido pela inoculação de fungos micorrízicos arbusculares.

5. Apesar de apresentar a menor colonização micorrízica, o estilosantes é a espécie com maior capacidade multiplicadora de esporos de fungos micorrízicos arbusculares no solo estudado.

\section{REFERÊNCIAS}

ALVES, G.L.N. Micorriza vesicular-arbuscular no crescimento e utilização do fósforo do solo pela braquiária e estilosantes. Lavras: UFLA, 1988. 42p. Dissertação de Mestrado.

BOTREL, M.A. Algumas considerações sobre gramíneas e leguminosas forrageiras. Coronel Pacheco: Embrapa-CNPGL, 1983. 51p. (EmbrapaCNPGL. Documentos, 9).

CARNEIRO, M.A.C.; SIQUEIRA, J.O.; VALE, F.R.; CURI, N. Limitação nutricional e efeito do pré-cultivo do solo com Brachiaria decumbens e da inoculação com Glomus etunicatum no crescimento de mudas de espécies arbóreas em solo degradado. Ciência e Prática, Lavras, v.19, n.3, p.281-288, jul./set. 1995.

CROWELL, M.F.; BOERNER, R.E.J. Influence of mycorrhizal and phosphorus on bellowground competition between two old-field annuals. Environment and Experimental Botany, Oxford, v.28, p.381-392, 1988.

FISHER, M.J.; RAO, I.M.; AYARZA, M.A.; LASCANO, C.E.; SANZ, J.I.; THOMAS, R.J.; VERA, R.R. Carbon storage by introduced deep-rooted grasses in the South American Savannas. Nature, London, v.371, n.15, p.236-238, Sept. 1994.

GERDEMANN, J.W.; NICOLSON, T.H. Spores of mycorrhizal endogone species extracted from soil by wet sieving and decanting. Transactions of the British Mycological Society, London, v.46, n.2, p.235-244, 1963.

GIOVANNETTI, M.; MOSSE, B. An evaluation of techniques to measure vesicular-arbuscular mycorrhizal infection in roots. New Phytologist, London, v.84, n.3, p.484-500, Mar. 1980. 
HUNTER, A.H. Laboratory analysis of vegetal tissues samples. Raleigh: International Soil Fertility Evaluation and Improvement Program, N.C.S.U., 1975. 5p. Normas de laboratório.

KORMANIK, P.P.; McGRAW, A.C. Quantification of vesicular-arbuscular mycorrhizal in plant roots. In: SCHENCK, N.C. (Ed.). Methods and principles of mycorrhizal research. St. Paul: American Phytopathological Society, 1982. p.37-46.

LAL, R.; STEWART, B.A. Soil degradation: a global threat. Advances in Soil Sciences, New York, v.14, p.289-330, 1989.

LAMBAIS, M.R. Condições edáficas que afetam o micotrofismo de Stylosanthes guianenesis (Aubl.) Sw. Piracicaba: ESALQ, 1987. 102p. Dissertação de Mestrado.

LUCHESI, L.A.C.; MORAES, A.; SANTOS, H.R.; SOUZA, M.L.P. Pastagens: um sistema de produção para reabilitação de áreas degradadas. In: SIMPÓSIO NACIONAL DE RECUPERAÇÃO DE ÁREAS DEGRADADAS, 1., 1992, Curitiba. Anais. Curitiba: UFPR, 1992. p.83-92.

MEDEIROS, J.C.; MIELNICZUK, J.; PEDO, F. Sistema de culturas adaptadas à produtividade, recuperação e conservação do solo. Revista Brasileira de Ciência do Solo, Campinas, v.1, n.2, p.199-204, abr. 1987.

MOSSE, B. Plant growth responses to vesiculararbuscular mycorrhiza. X. Responses of Stylosanthes and maize to inoculation in unsterile soil. New Phytologist, Oxford, v.78, p.277-288, 1977.

RAIJ, B. van. Fertilidade do solo e adubação. Piracicaba: Ceres/Potafos, 1991. 343p.

SAIF, S.R. Growth responses of tropical forage plant species to vesicular-arbuscular mycorrhizae. Plant and Soil, Dordrecht, v.97, p.25-35, 1987.
SARRUGE, J.R.; HAAG, H.P. Análises químicas em plantas. Piracicaba: USP-ESALQ, 1974. 56p.

SCHENCK, N.C.; PÉREZ, Y. Manual for the identification of VA mycorrhizal fungi. Gainesville: INVAM, University of Florida, 1987. $245 \mathrm{p}$.

SIMS, G.K. Biological degradation of soil. Advances in Soil Sciences, New York, v.14, p 331-339, 1989.

SIQUEIRA, J.O. Micorrizas arbusculares. In: ARAUJO, R.S.; HUNGRIA, M. (Eds.). Microrganismos de importância agrícola. Brasília: Embrapa, 1994. p.151-194.

SIQUEIRA, J.O.; COLOZZI FILHO, A.; OLIVEIRA, E. Ocorrência de micorrizas vesicular-arbusculares em agro e ecossistemas do Estado de Minas Gerais. Pesquisa Agropecuária Brasileira, Brasília, v.24, n.12, p.1499-1506, 1989.

SIQUEIRA, J.O.; MAHMUD, A.W.; HUBBELL, D.H. Comportamento diferenciado de fungos formadores de micorrizas vesicular-arbusculares em relação à acidez do solo. Revista Brasileira de Ciência do Solo, Campinas, v.10, p.11-16, 1986.

SIQUEIRA, J.O; MOREIRA, F.M.S.; GRISI, B.M.; HUNGRIA, M.; ARAUJO, R.S. Microrganismos e processos biológicos do solo: perspectiva ambiental. Brasília: Embrapa, 1994. 142p.

SIQUEIRA, J.O.; SAGGIN-JUNIOR, O. The importance of mycorrhizae association in natural in low fertility soils. In: SYMPOSIUM ON ENVIRONMENTAL STRESS: MAIZE IN PERSPECTIVE, 1., 1995, Sete Lagoas. Proceedings. Sete Lagoas: Embrapa, 1995. p.240-280.

ZONTA, E.P.; MACHADO, A.A.; SILVEIRA-JUNIOR, P. Sistemas de Análises Estatísticas para Microcomputadores (SANEST). Pelotas: UFPel, Dep. Mat. e Estatística, 1984. 151p.

Pesq. agropec. bras., Brasília, v.34, n.9, p.1669-1677, set. 1999 